\title{
Stability of solution of a backward problem of a time-fractional diffusion equation with perturbed order
}

\author{
Nguyen Minh Dien ${ }^{1,2}, *$, Dang Duc Trong ${ }^{1}$
}

${ }^{1}$ Faculty of Math and Computer Science, University of Science, VNU-HCM

${ }^{2}$ Thu Dau Mot University, Faculty of Natural Sciences

\section{Correspondence}

Nguyen Minh Dien, Faculty of Math and Computer Science, University of Science, VNU-HCM

Thu Dau Mot University, Faculty of Natural Sciences

Email: diennm@tdmu.edu.vn

\section{History}

- Received: 2018-12-03

- Accepted: 2019-03-19

- Published: 2019-03-29

DOI :

https://doi.org/10.32508/stdj.v22i1.1222

\section{Check for updates}

\section{Copyright}

(-) VNU-HCM Press. This is an openaccess article distributed under the terms of the Creative Commons Attribution 4.0 International license.

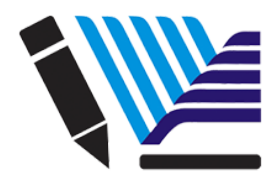

VNU-HCM Press

\begin{abstract}
The aim of this paper is of studying the stability of solution of a backward problem of a timefractional diffusion equation with perturbed order. We investigate the well-posedness of the backward problem with perturbed order for $t>0$. The results on the unique existence and continuity with respect to the fractional order, the source term as well as the final value of the solution are given. At $\mathrm{t}=0$ the backward problem is ill-posed and we introduce a truncated method to regularize the backward problem with respect to inexact fractional order. Some error estimates are provided in Holder type.
\end{abstract}

Key words: Caputo fractional derivative, stability of solution, ill-posed, regularization

\section{INTRODUCTION}

Let $\mathrm{T}>0, \quad \alpha \in(0,1), \quad \Omega=(0 ; \pi)$ and be the standard Laplace operator, we consider the inhomogeneous time-fractional diffusion equation

$$
\begin{cases}\mathrm{D}_{\mathrm{t}}^{\alpha} \mathrm{u}=\Delta \mathrm{u}+\mathrm{f}(\mathrm{x}, \mathrm{t}), & (\mathrm{x}, \mathrm{t}) \in \Omega \times(0, \mathrm{~T}), \quad \alpha \in(0,1) \\ \mathrm{u}(0, \mathrm{t})=\mathrm{u}(\pi, \mathrm{t})=0, & \\ \mathrm{u}(\mathrm{x}, \mathrm{T})=\mathrm{g}(\mathrm{x}) & \mathrm{x} \in \Omega,\end{cases}
$$

where $D_{t}^{\alpha}($.$) is the Caputo fractional derivative with respect to t$ of the order define as $\mathrm{D}_{\mathrm{t}}^{\alpha} \mathrm{u}(\mathrm{x}, \mathrm{t})= \begin{cases}\frac{1}{\Gamma(1-\alpha)} \int_{0}^{\mathrm{t}}(\mathrm{t}-\tau)^{-\alpha} \mathrm{u}_{\tau}(\mathrm{x}, \tau) \mathrm{d} \tau, & 0<\alpha<1 \\ \mathrm{u}_{\mathrm{t}}(\mathrm{x}, \mathrm{t}), & \alpha=1\end{cases}$ As is known, when $\alpha=1$ the problem (1.1) - (1.3) is ill-posed for any $0 \leq \mathrm{t}<$ Tand which was studied in many papers such as ${ }^{1,2}$. In the last decade, the fractional backward problem with $0<\alpha<1$ was investigated. In this case, the fractional linear backward problem is stable for $0<\mathrm{t}<\mathrm{T}$ and instable at $t=0$ which is differential from the case. Hence, regularization of solution at is in order. Ting Wei et al. ${ }^{3}$ and Tuan et al. ${ }^{4}$ used the Tikhonov method to regularizing the homogeneous and nonhomogeneous problem. Yang et al. ${ }^{5}$ also regularize the nonhomogeneous problem by the quasi-reversibility method. These papers used spectral method to obtain an explicit formula for the solution and gave regularization directly on that formula.

In the listed paper, the fractional order is assume to be known exactly. But in the real world problem, the parameter is defined by experiments. Hence, we only know its values inexactly. Even if the parameters are known exactly but are irrational, then we only have its approximate values to compute. Thus, a natural question that arises in numerical computing is whether the solution of a problem is stable with such approximate parameters. To the best of our knowledge, this question has still not been considered much. We can list here some papers. $\mathrm{Li}$ and Yamamoto ${ }^{6}$ investigated the solution of a forward problem with Neumann condition. Trong et al. ${ }^{2}$ studied the continuity of solutions of some linear fractional PDEs with perturbed orders. In our knowledge, until now, we do not find another paper which considers the backward problem with respect to the inexact order.

Base on the discussion above, we will

1. prove the well-posedness of the problem (1.1) - (1.3) when $0<\mathrm{t}<\mathrm{T}$ with respect to perturbed order.

2. regularization for the problem (1.1) - (1.3) at $t=0$ with the inexact order. 
The remainder of the present paper is organized as follows. The second section provides mathematical preliminaries, notations and lemmas which are used throughout the rest of this paper. In the third section, we investigate for the well-posedness of the problem (1.1) - (1.3) when $0<\mathrm{t}<\mathrm{T}$. Lastly, we give a method to regularization the problem (1.1) - (1.3) at $t=0$

\section{MATHEMATICAL PRELIMINARIES}

In this section we set up some notations and some Lemma which use to proof the main results of the paper. First, we list some properties of the Mittag-Leffler function

$\mathrm{E}_{\alpha, \beta}(\mathrm{z})=\sum_{\mathrm{k}=0}^{+\infty} \frac{\mathrm{z}^{\mathrm{k}}}{\Gamma(\mathrm{k} \alpha+\beta)}, \quad \mathrm{z} \in \mathrm{C}$

where $\alpha, \beta \in \mathrm{C}$ and $\operatorname{Re}(\alpha)>0$. For short, we also denote $\mathrm{E}_{\alpha, 1}(\mathrm{z})=\mathrm{E}_{\alpha}(\mathrm{z})$

Lemma $2.1^{7}$ Letting $\alpha, \lambda>0$ and $\mathrm{k} \in \mathrm{N}$, we have

$$
\frac{\mathrm{d}^{\mathrm{k}}}{\mathrm{dt}^{\mathrm{k}}} \mathrm{E}_{\alpha}\left(-\lambda \mathrm{t}^{\alpha}\right)=-\lambda \mathrm{t}^{\alpha-\mathrm{k}} \mathrm{E}_{\alpha, \alpha-\mathrm{k}+1}\left(-\lambda \mathrm{t}^{\alpha}\right), \quad \mathrm{t} \geq 0
$$

Lemma 2.2 $\left({ }^{8}\right.$ Let $0<\alpha_{*}<\alpha^{*}<1$ and let $\alpha, \alpha^{\prime} \in\left[\alpha_{*}, \alpha^{*}\right]$ then there exists a constant $C>0$ which dependent only on $\alpha_{*}, \alpha^{*}$ such that

$$
\begin{array}{ll}
\text { (i.) } & \frac{\mathrm{C}_{1}}{1+\lambda} \leq \mathrm{E}_{\alpha}(-\lambda) \leq \frac{\mathrm{C}_{2}}{1+\lambda}, \quad \forall \lambda \geq 0 \\
\text { (ii.) } \quad 0<\mathrm{E}_{\alpha}(-\lambda), \mathrm{E}_{\alpha, \alpha}(-\lambda) \leq \mathrm{C}, \quad \forall \lambda \geq 0 \\
\text { (iii.) }\left|\mathrm{E}_{\alpha}\left(-\mathrm{t}^{\alpha}\right)-\mathrm{E}_{\alpha^{\prime}}\left(-\mathrm{t}^{\alpha^{\prime}}\right)\right| \leq \mathrm{C}\left|\alpha-\alpha^{\prime}\right| \quad \mathrm{t} \geq 0 \\
\text { (iv.) }\left|\mathrm{E}_{\alpha}\left(-\lambda \mathrm{t}^{\alpha}\right)-\mathrm{E}_{\alpha^{\prime}}\left(-\lambda \mathrm{t}^{\alpha^{\prime}}\right)\right| \leq \mathrm{C} \lambda \ln \lambda\left|\alpha-\alpha^{\prime}\right| \quad \mathrm{t} \geq 0, \lambda>1 \\
\text { (v.) } \int_{0}^{t}\left|\mathrm{Q}(\alpha, \mathrm{t}, \tau)-\mathrm{Q}\left(\alpha^{\prime}, \mathrm{t}, \tau\right)\right| \mathrm{d} \tau \leq \mathrm{C} \lambda\left|\alpha-\alpha^{\prime}\right|, \quad \lambda>\lambda_{0}>0
\end{array}
$$

where $\mathrm{Q}(\mathrm{a}, \mathrm{t}, \tau)=(\mathrm{t}-\tau)^{\mathrm{a}-1} \mathrm{E}_{\mathrm{a}}\left(-\lambda(\mathrm{t}-\tau)^{\mathrm{a}}\right)$.

\section{THE WELL-POSEDNESS OF THE BACKWARD PROBLEM WITH $t>0$}

In this section, we give a condition to the backward problem have a unique solution and we also prove that the solution is dependent continuously on the fractional order and the final data.

As is known, by Fourier series the problem (1.1)-(1.2) corresponding to the initial data $\mathrm{u}(\mathrm{x}, 0)=\xi(\mathrm{x})$ can be transform to the integral equation as follows

$$
\mathrm{u}(\mathrm{x}, \mathrm{t})=\sum_{\mathrm{k}=1}^{+\infty}\left(\mathrm{E}_{\alpha}\left(-\lambda_{\mathrm{k}} \mathrm{t}^{\alpha}\right) \xi_{\mathrm{k}}+\int_{0}^{\mathrm{t}}(\mathrm{t}-\tau)^{\alpha-1} \mathrm{E}_{\alpha, \alpha}\left(-\lambda_{\mathrm{k}}(\mathrm{t}-\tau)^{\alpha}\right) \mathrm{f}_{\mathrm{k}}(\tau) \mathrm{d} \tau\right) \Phi_{\mathrm{k}}(\mathrm{x})
$$

Letting $t=T$ and then by direct computation, we obtain

$$
\mathrm{u}(\mathrm{x}, \mathrm{t})=\sum_{\mathrm{k}=1}^{+\infty}\left(\frac{\mathrm{E}_{\alpha}\left(-\lambda_{\mathrm{k}} \mathrm{t}^{\alpha}\right)}{\mathrm{E}_{\alpha}\left(-\lambda_{\mathrm{k}} \mathrm{T}^{\alpha}\right)} \mathrm{G}_{\mathrm{k}, \mathrm{f}, \mathrm{g}, \alpha}+\mathrm{H}_{\mathrm{k}, \mathrm{f}, \alpha}(\mathrm{t})\right) \Phi_{\mathrm{k}}(\mathrm{x})
$$

where $\mathrm{H}_{\mathrm{k}, \mathrm{f}, \alpha}(\mathrm{t})=\int_{0}^{\mathrm{t}}(\mathrm{t}-\tau)^{\alpha-1} \mathrm{E}_{\alpha, \alpha}\left(-\lambda_{\mathrm{k}}(\mathrm{t}-\tau)^{\alpha}\right) \mathrm{f}_{\mathrm{k}}(\tau) \mathrm{d} \tau, \quad \mathrm{G}_{\mathrm{kf}, g, \alpha}=\mathrm{g}_{\mathrm{k}}-\mathrm{H}_{\mathrm{k}, \alpha, \alpha}(\mathrm{T})$

Put

$$
\mathrm{G}_{\mathrm{f}, \mathrm{g}, \alpha}=\sum_{\mathrm{k}=1}^{+\infty} \mathrm{G}_{\mathrm{k}, \mathrm{f}, \alpha} \Phi_{\mathrm{k}}(\mathrm{x}), \quad \mathrm{H}_{\mathrm{f}, \alpha}(\mathrm{t})=\sum_{\mathrm{k}=1}^{+\infty} \mathrm{H}_{\mathrm{k}, \mathrm{f}, \alpha}(\mathrm{t}) \Phi_{\mathrm{k}}(\mathrm{x}) .
$$

From now on, we denote the solution of the backward problem (1.1)-(1.3) which satisfy (3.1) by $u_{\alpha, g, f}$ to emphasize the relationship of function $u$ with the data $\alpha, g, f$ In the following lemma, we give some estimates for $G_{f, \alpha}, H_{f, \alpha}(t)$.

Lemma 3.1. Let $\alpha \in(0,1)$ Let $g$ be the final data such that $g \in \mathrm{H}^{\mathrm{r}}(\Omega)$ and the source function $\mathrm{f} \in \mathrm{L}^{\infty}\left(0, \mathrm{~T} ; \mathrm{H}^{\mathrm{r}}(\Omega)\right)$ then we have

$$
\left\|\mathrm{H}_{\mathrm{f}, \alpha}(\mathrm{t})\right\|_{\mathrm{r}} \leq \sqrt{\mathrm{M}}\|\mathrm{f}\|_{\mathrm{L}^{\infty}\left(0, \mathrm{~T} ; \mathrm{H}^{\mathrm{r}}(\Omega)\right)}
$$




$$
\left\|\mathrm{G}_{\mathrm{f}, \mathrm{g}, \alpha}\right\|_{\mathrm{r}} \leq \sqrt{2}\left(\|\mathrm{~g}\|_{\mathrm{r}}+\sqrt{\mathrm{M}}\|\mathrm{f}\|_{\mathrm{L}^{\infty}\left(0, \mathrm{~T} ; \mathrm{H}^{r}(\Omega)\right)}\right)
$$

where $\mathrm{M}=\sum_{\mathrm{k}=1}^{+\infty} \frac{1}{\lambda_{\mathrm{k}}^{2}}$.

Proof. We have $\lambda_{\mathrm{k}}^{\mathrm{r}} \mathrm{f}_{\mathrm{k}} \leq \sum_{\mathrm{k}=1}^{+\infty} \lambda_{\mathrm{k}}^{\mathrm{r}} \mathrm{f}_{\mathrm{k}} \leq\|\mathrm{f}\|_{\mathrm{L}^{\infty}\left(0, \mathrm{~T} ; \mathrm{H}^{\mathrm{r}}(\Omega)\right)}$, which deduces that

$$
\begin{aligned}
\lambda_{\mathrm{k}}^{\mathrm{r}}\left|\mathrm{H}_{\mathrm{k}, \mathrm{f}, \alpha}(\mathrm{t})\right| & \leq \int_{0}^{\mathrm{t}}(\mathrm{t}-\tau)^{\alpha-1} \mathrm{E}_{\alpha, \alpha}\left(-\lambda_{\mathrm{k}}(\mathrm{t}-\tau)^{\alpha}\right) \lambda_{\mathrm{k}} \mathrm{f}_{\mathrm{k}}(\tau) \mathrm{d} \tau \\
& \leq\|\mathrm{f}\|_{\mathrm{L}^{\infty}\left(0, \mathrm{~T} ; \mathrm{H}^{\mathrm{r}}(\Omega)\right)} \int_{0}^{\mathrm{t}}(\mathrm{t}-\tau)^{\alpha-1} \mathrm{E}_{\alpha, \alpha}\left(-\lambda_{\mathrm{k}}(\mathrm{t}-\tau)^{\alpha}\right) \mathrm{d} \tau \\
& \left.\leq \frac{1}{\lambda_{\mathrm{k}}}\|\mathrm{f}\|_{\mathrm{L}^{\infty}\left(0, \mathrm{~T} ; \mathrm{H}^{\mathrm{r}}(\Omega)\right.}\right)
\end{aligned}
$$

due to the Lemma 1 we have $\int_{0}^{\mathrm{t}}(\mathrm{t}-\tau)^{\alpha-1} \mathrm{E}_{\alpha, \alpha}\left(-\lambda_{\mathrm{k}}(\mathrm{t}-\tau)^{\alpha}\right) \mathrm{d} \tau=\frac{1-\mathrm{E}_{\alpha}\left(-\lambda_{\mathrm{k}} \mathrm{t}^{\alpha}\right)}{\lambda_{\mathrm{k}}} \leq \frac{1}{\lambda_{\mathrm{k}}}$. The latter inequality yields

$$
\left\|\mathrm{H}_{\mathrm{f}, \alpha}(\mathrm{t})\right\|_{\mathrm{r}}^{2}=\sum_{\mathrm{k}=1}^{+\infty} \lambda_{\mathrm{k}}^{\mathrm{r}} \mathrm{H}_{\mathrm{k}, \mathrm{f}, \alpha}^{2}(\mathrm{t}) \leq\|\mathrm{f}\|_{\mathrm{L}^{\infty}\left(0, \mathrm{~T} ; \mathrm{T}^{\mathrm{r}}(\Omega)\right)} \sum_{\mathrm{k}=1}^{+\infty} \frac{1}{\lambda_{\mathrm{k}}^{2}}=\mathrm{M}\|\mathrm{f}\|_{\mathrm{L}^{\infty}\left(0, \mathrm{~T} ; \mathrm{H}^{\mathrm{r}}(\Omega)\right)}^{2} .
$$

This implies the inequality (3.2). To prove the inequality (3.3), we note that

$$
\left|\mathrm{G}_{\mathrm{k}, \mathrm{f}, \mathrm{g}, \alpha}\right|^{2} \leq 2\left(\left|\mathrm{~g}_{\mathrm{k}}\right|^{2}+\left|\mathrm{H}_{\mathrm{k}, \mathrm{f}, \alpha}(\mathrm{T})\right|^{2}\right),
$$

this follows

$$
\left\|\mathrm{G}_{\mathrm{f}, \mathrm{g}, \alpha}\right\|_{\mathrm{r}}^{2} \leq 2\left(\|\mathrm{~g}\|_{\mathrm{r}}^{2}+\mathrm{M}\|\mathrm{f}\|_{\mathrm{L}^{\infty}\left(0, \mathrm{~T} ; \mathrm{H}^{\mathrm{r}}(\Omega)\right)}^{2}\right) \leq 2\left(\|\mathrm{~g}\|_{\mathrm{r}}+\sqrt{\mathrm{M}}\|\mathrm{f}\|_{\mathrm{L}^{\infty}\left(0, \mathrm{~T} ; \mathrm{H}^{\mathrm{r}}(\Omega)\right)}\right)^{2} .
$$

This completed the proof of the Lemma.

Theorem 3.2. (Well-posedness) Let $\alpha \in(0,1)$ Let $g$ be the final data such that $g \in \mathrm{H}^{\mathrm{r}}(\Omega)$ and the source function $\mathrm{f} \in \mathrm{L}^{\infty}\left(0, \mathrm{~T} ; \mathrm{H}^{\mathrm{r}}(\Omega)\right)$. Then we have (i). If $r=0$ then the problem (1.1)-(1.3) has a unique solution

$$
\mathrm{u} \in \mathrm{L}^{2}\left(0, \mathrm{~T} ; \mathrm{H}_{0}^{1}(\Omega) \cap \mathrm{H}^{2}(\Omega)\right)
$$

which is given by

$$
\mathrm{u}(\mathrm{x}, \mathrm{t})=\sum_{\mathrm{k}=1}^{+\infty}\left(\frac{\mathrm{E}_{\alpha}\left(-\lambda_{\mathrm{k}} \mathrm{t}^{\alpha}\right)}{\mathrm{E}_{\alpha}\left(-\lambda_{\mathrm{k}} \mathrm{T}^{\alpha}\right)} \mathrm{G}_{\mathrm{k}, \mathrm{f}, \mathrm{g}, \alpha}+\mathrm{H}_{\mathrm{k}, \mathrm{f}, \alpha}(\mathrm{t})\right) \Phi_{\mathrm{k}}(\mathrm{x})
$$

where $\mathrm{G}_{\mathrm{k}, \mathrm{f}, \mathrm{g}, \alpha}, \mathrm{H}_{\mathrm{k}, \mathrm{f}, \alpha}(\mathrm{t})$ are defined in (4.1). Moreover, if $r=2$ then the problem (1.1)-(1.3) has a unique solution

$$
\mathrm{u} \in \mathrm{C}\left([0, \mathrm{~T}] ; \mathrm{L}^{2}(\Omega)\right) \cap \mathrm{C}\left((0, \mathrm{~T}) ; \mathrm{H}_{0}^{1}(\Omega) \cap \mathrm{H}^{2}(\Omega)\right)
$$

(ii). If $r>0$ then, for any $t>0$ we have

$$
\left\|\mathrm{u}_{\alpha, \mathrm{g}, \mathrm{f}}(., \mathrm{t})-\mathrm{u}_{\alpha^{\prime}, \mathrm{g}^{\prime}, \mathrm{f}^{\prime}}(., \mathrm{t})\right\| \leq \mathrm{Ct}^{-2 \alpha^{*}}\left(\left\|\mathrm{~g}-\mathrm{g}^{\prime}\right\|_{\mathrm{r}}^{2}+\left\|\mathrm{f}-\mathrm{f}^{\prime}\right\|_{\mathrm{L}^{\infty}\left(0, \mathrm{~T} ; \mathrm{H}^{\mathrm{r}}(\Omega)\right)}^{2}+\left|\alpha-\alpha^{\prime}\right|^{\frac{2}{4+\mathrm{r}}}\right)
$$

where C independent of $\left|\alpha-\alpha^{\prime}\right|,\left|\mathrm{g}-\mathrm{g}^{\prime}\right|,\left|\mathrm{f}-\mathrm{f}^{\prime}\right|$

Proof.

(i). The proof of Part (i) can be found in ${ }^{4}$.

(ii). The proof is subdivided into two steps.

- Step 1: $\left\|\mathrm{u}_{\alpha, \mathrm{g}, \mathrm{f}}(., \mathrm{t})-\mathrm{u}_{\alpha^{\prime}, \mathrm{g}, \mathrm{f}}(., \mathrm{t})\right\|^{2} \leq \mathrm{C}_{1} \mathrm{t}^{-2 \alpha^{*}}\left(\lambda_{\mathrm{p}}^{8}|\alpha-\alpha|^{2}+\lambda_{\mathrm{p}}^{-2 \mathrm{r}}\right)$

- Step 2: $\left\|\mathrm{u}_{\alpha^{\prime}, \mathrm{g}, \mathrm{f}}(., \mathrm{t})-\mathrm{u}_{\alpha^{\prime}, \mathrm{g}^{\prime}, \mathrm{f}^{\prime}}(., \mathrm{t})\right\|^{2} \leq \mathrm{C}_{2} \mathrm{t}^{-2 \alpha^{*}}\left(\left\|\mathrm{~g}-\mathrm{g}^{\prime}\right\|_{\mathrm{r}}^{2}+\left\|\mathrm{f}-\mathrm{f}^{\prime}\right\|_{\mathrm{L}^{\infty}\left(0, \mathrm{~T} ; \mathrm{H}^{\mathrm{r}}(\Omega)\right)}^{2}\right)$. 
Using the triangle inequality and combining Step 1 with Step 2 we obtain the desired. Indeed, from Step 1 and Step 2, we choose $p$ such that $p=\left[\left|\alpha-\alpha^{\prime}\right| \frac{1}{8+2 r}\right]+1$, then

$$
\begin{aligned}
& \left\|\mathrm{u}_{\alpha^{\prime}, \mathrm{g}, \mathrm{f}}(., \mathrm{t})-\mathrm{u}_{\alpha^{\prime}, \mathrm{g}^{\prime}, \mathrm{f}^{\prime}}(., \mathrm{t})\right\|^{2} \\
& \leq \mathrm{Ct}^{-2 \alpha^{*}}\left(\left\|\mathrm{~g}-\mathrm{g}^{\prime}\right\|_{\mathrm{r}}^{2}+\left\|\mathrm{f}-\mathrm{f}^{\prime}\right\|_{\mathrm{L}^{\infty}\left(0, \mathrm{~T} ; \mathrm{H}^{\mathrm{r}}(\Omega)\right)}^{2}+\lambda_{\mathrm{p}}^{8}\left|\alpha-\alpha^{\prime}\right|^{2}+\lambda_{\mathrm{p}}^{-2 \mathrm{r}}\right) \\
& \leq \mathrm{Ct}^{-2 \alpha^{*}}\left(\left\|\mathrm{~g}-\mathrm{g}^{\prime}\right\|_{\mathrm{r}}^{2}+\|\mathrm{f}-\mathrm{f}\|_{\mathrm{L}^{\infty}\left(0, \mathrm{~T} ; \mathrm{H}^{\mathrm{r}}(\Omega)\right)}^{2}+|\alpha-\alpha|^{\frac{2}{4+\mathrm{r}}}\right)
\end{aligned}
$$

Therefore, we only prove Step 1 and Step 2 in detail.

The proof of Step 1. Using the Cauchy-Schwarz inequality, we have

$$
\left\|\mathrm{u}_{\alpha, \mathrm{g}, \mathrm{f}}(., \mathrm{t})-\mathrm{u}_{\alpha^{\prime}, \mathrm{g}, \mathrm{f}}(., \mathrm{t})\right\|^{2} \leq 2 \mathrm{I}_{1}(\mathrm{t})+4\left(\mathrm{I}_{2}+\mathrm{I}_{3}\right)
$$

where

$$
\begin{aligned}
& \mathrm{I}_{1}(\mathrm{t})=\sum_{\mathrm{k}=1}^{+\infty}\left|\mathrm{H}_{\mathrm{k}, \mathrm{f}, \alpha}(\mathrm{t})-\mathrm{H}_{\mathrm{k}, \mathrm{f}, \alpha^{\prime}}(\mathrm{t})\right|^{2}=\left\|\mathrm{H}_{\mathrm{k}, \mathrm{f}, \alpha}(., \mathrm{t})-\mathrm{H}_{\mathrm{k}, \mathrm{f}, \alpha^{\prime}}(., \mathrm{t})\right\|^{2} \\
& \mathrm{I}_{2}=\sum_{\mathrm{k}=1}^{+\infty}\left(\frac{\mathrm{E}_{\alpha}\left(-\lambda_{\mathrm{k}} \mathrm{t}^{\alpha}\right)}{\mathrm{E}_{\alpha}\left(-\lambda_{\mathrm{k}} \mathrm{T}^{\alpha}\right)}\right)^{2}\left|\mathrm{G}_{\mathrm{k}, \mathrm{f}, \mathrm{g}, \alpha}-\mathrm{G}_{\mathrm{k}, \mathrm{f}, \mathrm{g}, \alpha^{\prime}}\right|^{2}, \\
& \mathrm{I}_{3}=\sum_{\mathrm{k}=1}^{+\infty}\left(\frac{\mathrm{E}_{\alpha}\left(-\lambda_{\mathrm{k}} \mathrm{t}^{\alpha}\right)}{\mathrm{E}_{\alpha}\left(-\lambda_{\mathrm{k}} \mathrm{T}^{\alpha}\right)}-\frac{\mathrm{E}_{\alpha}\left(-\lambda_{\mathrm{k}} \mathrm{t}^{\alpha^{\prime}}\right)}{\mathrm{E}_{\alpha^{\prime}}\left(-\lambda_{\mathrm{k}} \mathrm{T}^{\alpha^{\prime}}\right)}\right)^{2} \mathrm{G}_{\mathrm{k}, \mathrm{f}, g, \alpha}^{2}
\end{aligned}
$$

and $\mathrm{H}_{\mathrm{k}, \mathrm{f}, \alpha}(\mathrm{t}), \mathrm{G}_{\mathrm{k}, \mathrm{f}, \alpha}$ are defined in (3.1).

Estimating for $\mathrm{I}_{1}$. We can use Lemma 2.2 to obtain

$$
\left|\mathrm{H}_{\mathrm{k}, \mathrm{f}, \alpha}(\mathrm{t})-\mathrm{H}_{\mathrm{k}, \mathrm{f}, \alpha^{\prime}}(\mathrm{t})\right| \leq \mathrm{C} \lambda_{\mathrm{k}}\left|\alpha-\alpha^{\prime}\right| \leq \mathrm{C} \lambda_{\mathrm{p}}\left|\alpha-\alpha^{\prime}\right|, \quad \forall \mathrm{k} \leq \mathrm{p}
$$

due to $\lambda_{\mathrm{k}} \geq \lambda_{1}$ for any $\mathrm{k} \in \mathrm{N}$, which imply that

$\mathrm{I}_{1}(\mathrm{t})$

$$
\begin{aligned}
& =\sum_{\mathrm{k}=1}^{\mathrm{p}}\left|\mathrm{H}_{\mathrm{k}, \mathrm{f}, \alpha}(\mathrm{t})-\mathrm{H}_{\mathrm{k}, \mathrm{f}, \alpha}(\mathrm{t})\right|^{2}+2 \sum_{\mathrm{k}=\mathrm{p}+1}^{+\infty}\left(\left|\mathrm{H}_{\mathrm{k}, \mathrm{f}, \alpha}(\mathrm{t})\right|^{2}+\left.\mathrm{H}_{\mathrm{k}, \mathrm{f}, \alpha^{\prime}}(\mathrm{t})\right|^{2}\right) \\
& \leq \mathrm{Cp} \lambda_{\mathrm{p}}^{2}\left|\alpha-\alpha^{\prime}\right|^{2}+\lambda_{\mathrm{p}}^{-2 \mathrm{r}} \sum_{\mathrm{k}=\mathrm{p}+1}^{+\infty} \lambda_{\mathrm{k}}^{2 \mathrm{r}}\left(\left|\mathrm{H}_{\mathrm{k}, \mathrm{f}, \alpha}(\mathrm{t})\right|^{2}+\left|\mathrm{H}_{\mathrm{k}, \mathrm{f}, \alpha^{\prime}}(\mathrm{t})\right|^{2}\right) \\
& \leq \mathrm{Cp} \lambda_{\mathrm{p}}^{2}\left|\alpha-\alpha^{\prime}\right|^{2}+\lambda_{\mathrm{p}}^{-2 \mathrm{r}}\left(\left\|\mathrm{H}_{\mathrm{k}, \mathrm{f}, \alpha}(., \mathrm{t})\right\|_{\mathrm{r}}^{2}+\left\|\mathrm{H}_{\mathrm{k}, \mathrm{f}, \alpha^{\prime}}(., \mathrm{t})\right\|_{\mathrm{r}}^{2}\right) \\
& :=\mathrm{C}_{1}\left(\mathrm{p} \lambda_{\mathrm{p}}^{2}\left|\alpha-\alpha^{\prime}\right|^{2}+\lambda_{\mathrm{p}}^{-2 \mathrm{r}}\right) .
\end{aligned}
$$

Estimating for $\mathrm{I}_{2}$. From the Lemma 2.2, we have

$$
0<\frac{\mathrm{E}_{\alpha}\left(-\lambda_{\mathrm{k}} \mathrm{t}^{\gamma}\right)}{\mathrm{E}_{\alpha}\left(-\lambda_{\mathrm{k}} \mathrm{T}^{\gamma}\right)} \leq \mathrm{A}_{1}\left(\frac{\mathrm{T}}{\mathrm{t}}\right)^{\gamma} \leq \mathrm{A}_{1}\left(\frac{\mathrm{T}}{\mathrm{t}}\right)^{\alpha^{*}}=\mathrm{A}_{2} \mathrm{t}^{-\alpha^{*}}, \forall \gamma \in\left(0, \alpha^{*}\right),
$$

where $\mathrm{A}_{1}, \mathrm{~A}_{2}$ are independent of $\alpha, \lambda_{k}$.

Since $\left|G_{k, f, g, \alpha}-G_{k, f, g, \alpha^{\prime}}\right|=\left|H_{k, f, \alpha}(T)-H_{k, f, \alpha^{\prime}}(T)\right|$, therefore, from (3.5) and (3.6), we obtain

$$
\mathrm{I}_{2} \leq \mathrm{A}_{2} \mathrm{t}^{-2 \alpha^{*}} \mathrm{I}_{1}(\mathrm{~T}) \leq \mathrm{C}_{2} \mathrm{t}^{-2 \alpha^{*}}\left(\mathrm{p} \lambda_{\mathrm{p}}^{2}\left|\alpha-\alpha^{\prime}\right|^{2}+\lambda_{\mathrm{p}}^{-2 \mathrm{r}}\right)
$$

Estimating for $\mathrm{I}_{3}$. From the Lemma 2.2, for any $p>1$ we have

$$
\begin{aligned}
& \left|\mathrm{E}_{\alpha}\left(-\lambda_{\mathrm{k}} \mathrm{t}^{\alpha}\right) \mathrm{E}_{\alpha^{\prime}}\left(-\lambda_{\mathrm{k}} \mathrm{T}^{\alpha^{\prime}}\right)-\mathrm{E}_{\alpha}\left(-\lambda_{\mathrm{k}} \mathrm{T}^{\alpha}\right) \mathrm{E}_{\alpha^{\prime}}\left(-\lambda_{\mathrm{k}} \mathrm{t}^{\alpha^{\prime}}\right)\right| \\
& \leq \mathrm{C}_{30}\left(\left|\mathrm{E}_{\alpha}\left(-\lambda_{\mathrm{k}} \mathrm{T}^{\alpha}\right)-\mathrm{E}_{\alpha^{\prime}}\left(-\lambda_{\mathrm{k}} \mathrm{T}^{\alpha^{\prime}}\right)\right|+\left|\mathrm{E}_{\alpha}\left(-\lambda_{\mathrm{k}} \mathrm{t}^{\alpha}\right)-\mathrm{E}_{\alpha^{\prime}}\left(-\lambda_{\mathrm{k}} \mathrm{t}^{\alpha^{\prime}}\right)\right|\right) \\
& \leq \mathrm{C}_{31} \lambda_{\mathrm{p}} \ln \lambda_{\mathrm{p}}\left|\alpha-\alpha^{\prime}\right| .
\end{aligned}
$$


where $C_{31}$ is independent of $\left|\alpha-\alpha^{\prime}\right|$ and $p$ Thus we get

$$
\begin{aligned}
& \left|\frac{E_{\alpha}\left(-\lambda_{k} t^{\alpha}\right)}{E_{\alpha}\left(-\lambda_{k} T^{\alpha}\right)}-\frac{E_{\alpha}\left(-\lambda_{k} t^{\alpha^{\prime}}\right)}{E_{\alpha^{\prime}}\left(-\lambda_{k} T^{\alpha^{\prime}}\right)}\right| \\
& =\left|\frac{\mathrm{E}_{\alpha}\left(-\lambda_{\mathrm{k}} \mathrm{t}^{\alpha}\right) \mathrm{E}_{\alpha^{\prime}}\left(-\lambda_{\mathrm{k}} \mathrm{T}^{\alpha^{\prime}}\right)-\mathrm{E}_{\alpha}\left(-\lambda_{\mathrm{k}} \mathrm{T}^{\alpha}\right) \mathrm{E}_{\alpha^{\prime}}\left(-\lambda_{\mathrm{k}} \mathrm{t}^{\alpha^{\prime}}\right)}{\mathrm{E}_{\alpha}\left(-\lambda_{\mathrm{k}} \mathrm{T}^{\alpha}\right) \mathrm{E}_{\alpha^{\prime}}\left(-\lambda_{\mathrm{k}} \mathrm{T}^{\alpha^{\prime}}\right)}\right| \\
& \leq \mathrm{C}_{32} \lambda_{\mathrm{p}}^{3} \ln \lambda_{\mathrm{p}}\left|\alpha-\alpha^{\prime}\right|
\end{aligned}
$$

Combining (3.6) with the latter inequalities, we deduce

$$
\begin{aligned}
\mathrm{I}_{3}= & \sum_{\mathrm{k}=1}^{\mathrm{p}}\left(\frac{\mathrm{E}_{\alpha}\left(-\lambda_{\mathrm{k}} \mathrm{t}^{\alpha}\right)}{\mathrm{E}_{\alpha}\left(-\lambda_{\mathrm{k}} \mathrm{T}^{\alpha}\right)}-\frac{\mathrm{E}_{\alpha}\left(-\lambda_{\mathrm{k}} \mathrm{t}^{\mathrm{a}^{\prime}}\right)}{\mathrm{E}_{\alpha^{\prime}}\left(-\lambda_{\mathrm{k}} \mathrm{T}^{\alpha^{\prime}}\right)}\right)^{2} \mathrm{G}_{\mathrm{k}, \mathrm{f}, g, \alpha}^{2} \\
& +\sum_{\mathrm{k}=\mathrm{p}+1}^{+\infty}\left(\frac{\mathrm{E}_{\alpha}\left(-\lambda_{\mathrm{k}} \mathrm{t}^{\alpha}\right)}{\mathrm{E}_{\alpha}\left(-\lambda_{\mathrm{k}} \mathrm{T}^{\alpha}\right)}-\frac{\mathrm{E}_{\alpha^{\prime}}\left(-\lambda_{\mathrm{k}} \mathrm{t}^{\alpha^{\prime}}\right)}{\mathrm{E}_{\alpha^{\prime}}\left(-\lambda_{\mathrm{k}} \mathrm{T}^{\alpha^{\prime}}\right)}\right)^{2} \mathrm{G}_{\mathrm{k}, \mathrm{f}, g, \alpha}^{2} \\
& \leq \mathrm{C}_{33}\left\|\mathrm{G}_{\mathrm{f}, \mathrm{g}, \alpha^{\prime}}\right\|^{2} \lambda_{\mathrm{p}}^{3} \ln \lambda_{\mathrm{p}}\left|\alpha-\alpha^{\prime}\right|+\mathrm{C}_{34} \mathrm{t}^{\alpha^{*}} \sum_{\mathrm{k}=\mathrm{p}+1}^{+\infty} \mathrm{G}_{\mathrm{k}, \mathrm{f}, \mathrm{g}, \alpha^{\prime}}^{2}
\end{aligned}
$$

Using Lemma 3.1, we have

$$
\begin{aligned}
& \mathrm{I}_{3} \\
& \leq \mathrm{C}_{35} \lambda_{\mathrm{p}}^{6} \ln \lambda_{\mathrm{p}}^{2}\left|\alpha-\alpha^{\prime}\right|^{2}+\mathrm{C}_{35} \lambda_{\mathrm{p}}^{-2 \mathrm{r}_{\mathrm{t}}-2 \alpha^{*}} \sum_{\mathrm{k}=\mathrm{p}+1}^{+\infty} \lambda_{\mathrm{k}}^{2 \mathrm{r}} \mathrm{G}_{\mathrm{k}, \mathrm{f}, \mathrm{g}, \alpha}^{2} \\
& \leq \mathrm{C}_{3}\left(\lambda_{\mathrm{p}}^{8}|\alpha-\alpha|^{2}+\lambda_{\mathrm{p}}^{-2 \mathrm{r}_{\mathrm{t}}-2 \alpha^{*}}\right)
\end{aligned}
$$

due to $\ln \lambda_{p} \leq \lambda_{p}$. Since $1 \leq p \leq \lambda_{p}$, then from (3.5), (3.7) and (3.8), we obtain

$$
\left\|\mathrm{u}_{\alpha, \mathrm{g}, \mathrm{f}}(., \mathrm{t})-\mathrm{u}_{\alpha^{\prime}, \mathrm{g}, \mathrm{f}}(., \mathrm{t})\right\|^{2} \leq \mathrm{C}_{1} \mathrm{t}^{-2 \alpha^{*}}\left(\lambda_{\mathrm{p}}^{8}|\alpha-\alpha|^{2}+\lambda_{\mathrm{p}}^{-2 \mathrm{r}}\right)
$$

This completed the proof of Step 1. We now proof Step 2.

The proof of Step 2.

$$
\begin{aligned}
& \left\|\mathrm{u}_{\alpha^{\prime}, \mathrm{g}, \mathrm{f}}(., \mathrm{t})-\mathrm{u}_{\alpha^{\prime}, \mathrm{g}^{\prime}, \mathrm{f}^{\prime}}(., \mathrm{t})\right\|^{2} \\
& \leq \sum_{\mathrm{k}=1}^{+\infty}\left(\frac{\mathrm{E}_{\alpha^{\prime}}\left(-\lambda_{\mathrm{k}} \mathrm{t}^{\alpha^{\prime}}\right)}{\mathrm{E}_{\alpha^{\prime}}\left(-\lambda_{\mathrm{k}} \mathrm{T}^{\alpha^{\prime}}\right)}\left(\mathrm{G}_{\mathrm{k}, \mathrm{f}, \mathrm{g}, \alpha^{\prime}}-\mathrm{G}_{\mathrm{k}, \mathrm{f}^{\prime}, \mathrm{g}^{\prime}, \alpha^{\prime}}\right)+\left(\mathrm{H}_{\mathrm{k}, \mathrm{f}, \mathrm{g}, \alpha^{\prime}}(\mathrm{t})-\mathrm{H}_{\mathrm{k}, \mathrm{f}^{\prime}, \mathrm{g}^{\prime}, \alpha^{\prime}}(\mathrm{t})\right)\right)^{2} \\
& \leq 2 \sum_{\mathrm{k}=1}^{+\infty}\left(\frac{\mathrm{E}_{\alpha^{\prime}}\left(-\lambda_{\mathrm{k}} \mathrm{t}^{\alpha^{\prime}}\right)}{\mathrm{E}_{\alpha^{\prime}}\left(-\lambda_{\mathrm{k}} \mathrm{T}^{\alpha^{\prime}}\right)}\left|\mathrm{G}_{\mathrm{k}, \mathrm{f}-\mathrm{f}^{\prime}, \mathrm{g}-\mathrm{g}^{\prime}, \alpha^{\prime}}\right|^{2}+\left|\mathrm{H}_{\mathrm{k}, \mathrm{f}-\mathrm{f}^{\prime}, \alpha^{\prime}}(\mathrm{t})\right|^{2}\right)^{2}
\end{aligned}
$$

We can use the Lemma 2.1 and (3.6) to obtain

$$
\begin{aligned}
& \left\|\mathrm{u}_{\alpha^{\prime}, \mathrm{g}, \mathrm{f}}(., \mathrm{t})-\mathrm{u}_{\alpha^{\prime}, \mathrm{g}^{\prime}, \mathrm{f}^{\prime}}(., \mathrm{t})\right\|^{2} \\
& \leq 2\left[\mathrm{C}_{36} \mathrm{t}^{-2 \alpha^{*}}\left(\left(\left\|\mathrm{~g}-\mathrm{g}^{\prime}\right\|_{\mathrm{r}}^{2}+\mathrm{M}\left\|\mathrm{f}-\mathrm{f}^{\prime}\right\|_{\mathrm{L}^{\infty}\left(0, \mathrm{~T} ; \mathrm{H}^{\mathrm{r}}(\Omega)\right)}\right)\right)+\mathrm{M}\left\|\mathrm{f}-\mathrm{f}^{\prime}\right\|_{\mathrm{L}^{\infty}\left(0, \mathrm{~T} ; \mathrm{H}^{\mathrm{r}}(\Omega)\right)}^{2}\right] \\
& \leq \mathrm{C}_{2} \mathrm{t}^{-2 \alpha^{*}}\left(\left(\left\|\mathrm{~g}-\mathrm{g}^{\prime}\right\|_{\mathrm{r}}^{2}+\left\|\mathrm{f}-\mathrm{f}^{\prime}\right\|_{\mathrm{L}^{\infty}\left(0, \mathrm{~T} ; \mathrm{H}^{\mathrm{r}}(\Omega)\right)}\right)\right) .
\end{aligned}
$$

This completed the proof of Step 2 and the proof of the Theorem. 
REGULARIZATION AND ERROR ESTIMATES FOR BACKWARD PROBLEM AT

\section{$t=0$}

In this section, we propose a regularization method to regularize solution of the backward problem at $\mathrm{t}=0$ we will give some error estimates in the case of inexact order.

Let $\varepsilon \in(0,1)$, and $\alpha_{\varepsilon} \in(0,1), \mathrm{g}_{\varepsilon} \in \mathrm{H}^{\mathrm{r}}(\Omega), \mathrm{f}_{\varepsilon} \in \mathrm{L}^{\infty}\left(0, \mathrm{~T} ; \mathrm{H}^{\mathrm{r}}(\Omega)\right)$ be measurement data such that the following condition

$$
\left|\alpha-\alpha_{\varepsilon}\right|<\varepsilon,\left\|\mathrm{g}-\mathrm{g}_{\varepsilon}\right\|_{\mathrm{r}}<\varepsilon,\left\|\mathrm{f}-\mathrm{f}_{\varepsilon}\right\|_{\mathrm{L}^{\infty}\left(0, \mathrm{~T} ; \mathrm{H}^{\mathrm{r}}(\Omega)\right)}<\varepsilon .
$$

We approximate the solution of the backward problem at $\mathrm{t}=0$ by the problem

$$
\mathrm{u}_{\alpha, \mathrm{f}, \mathrm{f}}^{\mathrm{p}}(\mathrm{x})=\sum_{\mathrm{k}=1}^{\mathrm{p}} \frac{\mathrm{G}_{\mathrm{k}, \mathrm{f}, \mathrm{g}, \alpha}}{\mathrm{E}_{\alpha}\left(-\lambda_{\mathrm{k}} \mathrm{T}^{\alpha}\right)^{\alpha}} \Phi_{\mathrm{k}}(\mathrm{x})
$$

where $p$ is the regularization parameter and $G_{k, f, g, \alpha}$ is defined in (3.1).

First, we prove that the problem (4.2) is well-posed with respect to the fractional order.

Theorem 4.1 Let $0<\alpha_{*}<\alpha^{*}<1$ and let $\alpha, \alpha_{\varepsilon} \in\left[\alpha_{*}, \alpha^{*}\right]$. Let $\mathrm{g}, \mathrm{g}_{\varepsilon} \in \mathrm{H}^{\mathrm{r}}(\Omega)$ and $\mathrm{f}, \mathrm{f}_{\varepsilon} \in \mathrm{L}^{\infty}\left(0, \mathrm{~T} ; \mathrm{H}^{\mathrm{r}}(\Omega)\right)$. Then we have

$$
\mathrm{u}_{\alpha, \mathrm{f}, \mathrm{g}}^{\mathrm{p}}(.)-\mathrm{u}_{\alpha_{\varepsilon}, \mathrm{f}_{\varepsilon}, \mathrm{g}_{\varepsilon}}^{\mathrm{p}}(.) \| \leq D \lambda_{\mathrm{p}}^{9 / 2}\left(\left|\alpha-\alpha_{\varepsilon}\right|+\left\|\mathrm{g}-\mathrm{g}_{\varepsilon}\right\|_{\mathrm{r}}+\left\|\mathrm{f}-\mathrm{f}_{\varepsilon}\right\|_{\mathrm{L}^{\infty}\left(0, \mathrm{~T} ; \mathrm{H}^{\mathrm{r}}(\Omega)\right)}\right),
$$

where $\mathrm{D}$ is independent of $\alpha-\alpha_{\varepsilon}, g-g_{\varepsilon}, f-f_{\varepsilon}$.

Proof. Using Lemma 2.2, we have

$$
\left|\frac{1}{\mathrm{E}_{\alpha}\left(-\lambda_{\mathrm{k}} \mathrm{T}^{\alpha}\right)}-\frac{1}{\mathrm{E}_{\alpha_{\mathrm{c}}}\left(-\lambda_{\mathrm{k}} \mathrm{T}^{\alpha_{\varepsilon}}\right)}\right| \leq \mathrm{C}_{43} \lambda_{\mathrm{p}}^{4}\left|\alpha-\alpha_{\varepsilon}\right|
$$

for any $k \leq p$. This follows that

$$
\left|\frac{1}{\mathrm{E}_{\alpha}\left(-\lambda_{\mathrm{k}} \mathrm{T}^{\alpha}\right)}-\frac{1}{\mathrm{E}_{\alpha_{\varepsilon}}\left(-\lambda_{\mathrm{k}} \mathrm{T}^{\alpha_{\varepsilon}}\right)}\right| \leq \mathrm{C}_{43} \lambda_{\mathrm{p}}^{4}\left|\alpha-\alpha_{\varepsilon}\right|,
$$

where $C_{43}$ is independent of $\alpha, \alpha_{\varepsilon}, p$.

Since

$$
\begin{aligned}
\left|\mathrm{H}_{\mathrm{k}, \mathrm{f}, \alpha}-\mathrm{H}_{\mathrm{k}, \mathrm{f}_{\mathrm{e}}, \alpha_{\varepsilon}}\right|^{2} & \leq 2\left(\left|\mathrm{H}_{\mathrm{k}, \mathrm{f}, \alpha}-\mathrm{H}_{\mathrm{k}, \mathrm{f}_{\varepsilon}, \alpha}\right|^{2}+\left|\mathrm{H}_{\mathrm{k}, \mathrm{f}, \alpha}-\mathrm{H}_{\mathrm{k}, \mathrm{f}_{e}, \alpha_{\varepsilon}}\right|^{2}\right) \\
& =2\left(\left|\mathrm{H}_{\mathrm{k}, \mathrm{f}-\mathrm{f}, \alpha}\right|^{2}+\left|\mathrm{H}_{\mathrm{k}, \mathrm{f}, \alpha}-\mathrm{H}_{\mathrm{k}, \mathrm{f}_{\varepsilon}, \alpha_{\varepsilon}}\right|^{2}\right)
\end{aligned}
$$

we can use the same method of estimating of (3.5) and Lemma 2.1 to get

$$
\begin{aligned}
& \sum_{\mathrm{k}=1}^{\mathrm{p}}\left|\mathrm{G}_{\mathrm{k}, \mathrm{f}, \mathrm{g}, \alpha}-\mathrm{G}_{\mathrm{k}, \mathrm{f}_{\varepsilon}, \mathrm{g}_{\varepsilon}, \alpha_{\varepsilon}}\right|^{2} \\
& \leq 4\left(\sum_{\mathrm{k}=1}^{\mathrm{p}}\left|\mathrm{g}_{\mathrm{k}}-\mathrm{g}_{\mathrm{ek}}\right|^{2}+\left|\mathrm{H}_{\mathrm{k}, \mathrm{f}-\mathrm{f}_{\varepsilon}, \alpha}\right|^{2}+\left|\mathrm{H}_{\mathrm{k}, \mathrm{f}, \alpha}-\mathrm{H}_{\mathrm{k}, \mathrm{f}_{\varepsilon}, \alpha_{\varepsilon}}\right|^{2}\right) \\
& \leq 4\left(\left\|\mathrm{~g}-\mathrm{g}_{\varepsilon}\right\|^{2}+\left\|\mathrm{f}-\mathrm{f}_{\varepsilon}\right\|^{2}+\mathrm{pC} \lambda_{\mathrm{p}}^{2}\left|\alpha-\alpha_{\varepsilon}\right|^{2}\right)
\end{aligned}
$$

where $C$ is independent of $\alpha, \alpha_{\varepsilon}, p$. We combine (4.3) and (4.4) to obtain

$$
\begin{aligned}
& \left\|\mathrm{u}_{\alpha, \mathrm{f}, \mathrm{g}}^{\mathrm{p}}(.)-\mathrm{u}_{\alpha_{\varepsilon}, \mathrm{f}_{\varepsilon}, \mathrm{g}_{\varepsilon}}^{\mathrm{p}}(.)\right\|^{2} \\
& \left.\leq\left. 2\left(\sum_{\mathrm{k}=1}^{\mathrm{p}}\left|\frac{\mathrm{G}_{\mathrm{k}, \mathrm{f}_{\varepsilon}, \mathrm{g}_{\varepsilon}, \alpha_{\varepsilon}}}{\mathrm{E}_{\alpha_{\varepsilon}}\left(-\lambda_{\mathrm{k}} \mathrm{T}_{\varepsilon}\right)}\right|^{2}+\sum_{\mathrm{k}=1}^{\mathrm{p}} \| \frac{1}{\mathrm{E}_{\alpha}\left(-\lambda_{\mathrm{k}} \mathrm{T}^{\alpha}\right)^{-}}-\frac{1}{\mathrm{E}_{\alpha_{\varepsilon}}\left(-\lambda_{\mathrm{k}} \mathrm{T}^{\alpha_{\varepsilon}}\right)}\right) \mathrm{G}_{\mathrm{k}, \mathrm{f}, \mathrm{g}, \alpha}\right|^{2}\right) \\
& \leq 2 \mathrm{C} \lambda_{\mathrm{p}}^{2}\left(\left\|\mathrm{~g}-\mathrm{g}_{\varepsilon}\right\|^{2}+\left\|\mathrm{f}-\mathrm{f}_{\varepsilon}\right\|^{2}+\mathrm{pC} \lambda_{\mathrm{p}}^{2}\left|\alpha-\alpha_{\varepsilon}\right|^{2}\right)+\mathrm{C}_{43}^{2} \mathrm{p} \lambda_{\mathrm{p}}^{8}\left|\alpha-\alpha_{\varepsilon}\right|^{2} \\
& \leq \mathrm{C}_{44} \lambda_{\mathrm{p}}^{9}\left(\left\|\mathrm{~g}-\mathrm{g}_{\varepsilon}\right\|_{\mathrm{H}^{\mathrm{r}}(\Omega)}+\left\|\mathrm{f}-\mathrm{f}_{\mathcal{\varepsilon}}\right\|_{\mathrm{L}^{\infty}\left(0, \mathrm{~T} ; \mathrm{H}^{\mathrm{r}}(\Omega)\right)}+\left|\alpha-\alpha_{\varepsilon}\right|\right)^{2}
\end{aligned}
$$


due to $p \leq \lambda_{p}$, where $C_{44}$ is independent of $\mathrm{g}-\mathrm{g}_{\varepsilon}, \alpha-\alpha_{\varepsilon}, \mathrm{p}$. This imply the result of the Theorem.

Theorem 4.2 Let $0<\alpha_{*}<\alpha^{*}<1$ and let $\alpha, \alpha_{\varepsilon} \in\left[\alpha_{*}, \alpha^{*}\right]$. Let $\mathrm{g}, \mathrm{g}_{\varepsilon} \in \mathrm{H}^{\mathrm{r}}(\Omega)$ and $\mathrm{f}, \mathrm{f}_{\varepsilon} \in \mathrm{L}^{\infty}\left(0, \mathrm{~T} ; \mathrm{H}^{\mathrm{r}}(\Omega)\right)$ be the measurement data which satisfy (4.1). We suppose further that $\| \mathrm{u}\left(.0 \|_{\mathrm{r}} \leq \mathrm{E}\right.$ Choose $\mathrm{p}=\left[\varepsilon^{\frac{1}{2 \mathrm{r}+9}}\right]+1$ then we have the following estimate

$$
\left\|\mathrm{u}_{\alpha, \mathrm{f}, \mathrm{g}}(.)-\mathrm{u}_{\alpha_{\varepsilon}, \mathrm{f}_{\varepsilon}, \mathrm{g}_{\varepsilon}}^{\mathrm{p}}(.)\right\| \leq \mathrm{Q} \varepsilon^{\frac{2 \mathrm{r}}{2 \mathrm{r}+9}}
$$

where $Q$ independent of $\varepsilon$

Proof. We have

$$
\left\|\mathrm{u}_{\alpha, \mathrm{f}, \mathrm{g}}(., 0)-\mathrm{u}_{\alpha, \mathrm{f}, \mathrm{g}}^{\mathrm{p}}(.)\right\|^{2}=\sum_{\mathrm{k}=\mathrm{p}+1}^{+\infty}\left|\frac{\mathrm{G}_{\mathrm{k}, \mathrm{f}, \mathrm{g}, \alpha}}{\mathrm{E}_{\alpha}\left(-\lambda_{\mathrm{k}} \mathrm{T}^{\alpha}\right)}\right|^{2} \leq \frac{1}{\lambda_{\mathrm{p}}^{\mathrm{r}}} \sum_{\mathrm{k}=\mathrm{p}+1}^{+\infty} \lambda_{\mathrm{p}}^{\mathrm{r}}\left|\mathrm{u}_{\mathrm{k}, \alpha, \mathrm{f}, \mathrm{g}}(., 0)\right|^{2} \leq \mathrm{E} \lambda_{\mathrm{p}}^{-\mathrm{r}}
$$

Using the triangle inequality, Theorem 4.1 and the latter inequality, we obtain

$$
\begin{aligned}
\left\|\mathrm{u}_{\alpha, \mathrm{f}, \mathrm{g}}(.)-\mathrm{u}_{\alpha_{\varepsilon}, \mathrm{f}_{\varepsilon}, \mathrm{g}_{\varepsilon}}^{\mathrm{p}}(.)\right\| & \leq\left\|\mathrm{u}_{\alpha, \mathrm{f}, \mathrm{g}}(.)-\mathrm{u}_{\alpha, \mathrm{f}, \mathrm{g}}^{\mathrm{p}}(.)\right\|+\left\|\mathrm{u}_{\alpha, \mathrm{f}, \mathrm{g}}^{\mathrm{p}}(.)-\mathrm{u}_{\alpha_{\varepsilon}, \mathrm{f}_{\varepsilon}, \mathrm{g}_{\varepsilon}}^{\mathrm{p}}(.)\right\| \\
& \leq \mathrm{E} \lambda_{\mathrm{p}}^{-\mathrm{r}}+\mathrm{D} \lambda_{\mathrm{p}}^{9 / 2}\left(\left|\alpha-\alpha_{\varepsilon}\right|+\left\|\mathrm{g}-\mathrm{g}_{\varepsilon}\right\|_{\mathrm{r}}+\left\|\mathrm{f}-\mathrm{f}_{\mathcal{E}}\right\|_{\mathrm{L}^{\infty}\left(0, \mathrm{~T} ; \mathrm{H}^{\mathrm{r}}(\Omega)\right)}\right) \\
& \leq \mathrm{Q}_{0}\left(\lambda_{\mathrm{p}}^{-\mathrm{r}}+\lambda_{\mathrm{p}}^{9 / 2} \varepsilon\right)
\end{aligned}
$$

where $\mathrm{Q}_{0}=\max \{\mathrm{E}, 3 \mathrm{D}\}$. Choose $\mathrm{p}=\left[\varepsilon^{\frac{1}{2 \mathrm{r}+9}}\right]+1$, and notice that $\lambda_{\mathrm{p}}=\mathrm{p}^{2}$, we obtain

$$
\left\|\mathrm{u}_{\alpha, \mathrm{f}, \mathrm{g}}(.)-\mathrm{u}_{\alpha_{\varepsilon}, \mathrm{f}_{\varepsilon}, \mathrm{g}_{\varepsilon}}^{\mathrm{p}}(.)\right\| \leq \mathrm{Q} \varepsilon^{\frac{2 \mathrm{r}}{2 \mathrm{r}+9}},
$$

where $Q$ is independent of $\varepsilon$ This completes the proof of the Theorem.

\section{CONCLUSIONS}

In this paper, we investigate a backward problem for a non-homogeneous a time-fractional diffusion equation. For the well-posed problem part, the unique existence and continuity with respect to the fractional order, the source term as well as the final value of the solution are given. For the ill-posed problem part, we propose the truncated method for obtaining a regularized solution. The convergence results obtained under the Holder type. In the future, we will consider the problem for a class of fractional equation with both time and space fractional order with linear and/or nonlinear source.

\section{COMPETING INTERESTS}

The authors declare that they have no conflicts of interest.

\section{AUTHORS' CONTRIBUTIONS}

Nguyen Minh Dien is a Ph.D. student of the University of Science (VNU-HCM) who wrote and revised this manuscript under the scientific guidance of Professor Dang Duc Trong.

\section{REFERENCES}

1. Trong DD, Tuan NH. Regularization and error estimates for a nonhomogeneous final value heat problem. Electronic Journal of Differential Equations;2006(4):1-10.

2. Trong DD, Tuan NH. Regularization and error estimates for a nonhomogeneous final value heat problem. Electronic Journal of Differential Equations. 2008;(33):1-14.

3. Wei T, Wang, Jun-Gang. A modified quasi-boundary value method for the final value time-fractional diffusion problem. ESAIM: M2AN. 2014;48(2):603-621.

4. Tuan NH, Long LD, Tatar S. Tikhonov regularization method for a backward problem for the inhomogeneous time-fractional diffusion equation. Applicable Analysis. Applicable Analysis. 2017;74(6):1340-1361. Available from: 10.1080/00036811.2017.1293815.

5. Yang F, Ren YP, Li XX. The quasi-reversibility method for a final value problem of the time-fractional diffusion equation with inhomogeneous source. Mathematical Methods in Applied Sciences. 2014;48:603-621. Available from: 10.1002/mma.4705.

6. Li G, Zhang D, Jia X, Yamamoto M. Simultaneous inversion for the space- dependent diffusion coefficient and the fractional order in the time-fractional diffusion equation. Inverse Problems. 2013;29(6):065014. Available from: 10.1088/0266-5611/29/6/065014.

7. Sakamoto K, Yamamoto M. Initial value/boundary value problems for fractional diffusion-wave equations and applications to some inverse problem. Journal of Mathematical Analysis and Applications. 2011;382(1):426-447. Available from: 10.1016/j.jmaa.2011.04.058.

8. Dang DT, Nane E, Nguyen DM, Tuan NH. Continuity of solutions of a class of fractional equations. Potential Analysis. 2018;49(3):423478. Available from: $10.1007 / \mathrm{s} 11118-017-9663-5$. 